\title{
Kolmogorovian Active Turbulence of a Sparse Assembly of Interacting Marangoni Surfers
}

\author{
Mickael Bourgoin $\odot,{ }^{1}$ Ronan Kervil, ${ }^{2}$ Cecile Cottin-Bizonne $\odot,{ }^{2}$ Florence Raynalø ${ }^{3}$ \\ Romain Volk@, ${ }^{1}$ and Christophe Ybert $\oplus^{2, *}$ \\ ${ }^{1}$ Laboratoire de Physique, Universite Lyon, ENS de Lyon, Universite Lyon 1, \\ CNRS, F-69342 Lyon, France \\ ${ }^{2}$ ILM, Universite Lyon, Universite Lyon 1, CNRS, F-69622 Villeurbanne CEDEX, France \\ ${ }^{3}$ LMFA, Université Lyon, Centrale Lyon, INSA Lyon, Université Lyon 1, CNRS, F-69134 Écully, France
}

(Received 13 November 2019; revised manuscript received 25 April 2020; accepted 4 May 2020; published 22 June 2020)

Active matter, composed of self-propelled entities, forms a wide class of out-of-equilibrium systems that display striking collective behaviors, among which, the so-called active turbulence where spatially and time-disordered flow patterns spontaneously arise in a variety of active systems. De facto, the active turbulence naming suggests a connection with a second seminal class of out-of-equilibrium systems, inertial turbulence, even though the latter is of very different nature with energy injected at global system scale rather than at the elementary scale of single constituents. Indeed, the existence of a possible strong tie between active and canonical turbulence remains an open question and a field of profuse research. Using an assembly of self-propelled interfacial particles, we show experimentally that the statistical properties of particles' velocities display a turbulentlike behavior, as described by the celebrated 1941 phenomenology of Kolmogorov. Moreover, the analogy between the dynamics of the self-propelled particles and inertial turbulence is observed to hold consistently both in the Eulerian and Lagrangian frameworks. Unlike the swimmers' velocities distribution, the subsurface fluid flow is found not turbulent, thus making Marangoni surfers' assemblies different from other active systems generating turbulence, such as living matter. Identifying an active system in the universality class of inertial turbulence not only benefits its future development but may also provide new insights into the long-standing description of turbulent flows, arguably one of the biggest remaining mysteries in classical physics.

DOI: 10.1103/PhysRevX.10.021065

\section{INTRODUCTION}

Active living organisms, such as bacterial suspensions, birds, fishes, etc., tend to self-organize (in swarms, schools, flocks, etc.) and develop coherent collective motions, with important consequences in terms of, for instance, nutrientfinding strategies or protection against predators [1-6]. Such systems share the feature of being intrinsically out of equilibrium, as energy is constantly injected at the level of each individual entity. This property makes their statistical modeling a conceptual challenge, as usual tools from statistical thermodynamics at equilibrium cannot be used. Interestingly, the emergence of large-scale collective dynamics while energy sources are at small scale, underlies

\footnotetext{
* Corresponding author. christophe.ybert@univ-lyon1.fr

Published by the American Physical Society under the terms of the Creative Commons Attribution 4.0 International license. Further distribution of this work must maintain attribution to the author(s) and the published article's title, journal citation, and DOI.
}

Subject Areas: Fluid Dynamics, Nonlinear Dynamics, Soft Matter the existence of multiscale correlations driven by interentities' interactions.

This scenario naturally resonates with the energy cascade phenomenology, the cornerstone of inertial turbulence description. A direct turbulent energy cascade describes the process where mechanical energy injected into a flow at some large-scale $L$ (e.g., by steering of shearing the fluid) flows down to smaller scales (generating intricate multiscale motions) until it is dissipated into heat by viscous friction at some small-scale $\eta$. Such a direct cascade drives the multiscale dynamics of 3D turbulence (2D turbulence exhibits an inverse cascade, where energy flows from small to large scales).

In 1941, Kolmogorov proposed a self-similar description of this process [7]. He predicted that there exists an inertial range of length scales in between a dissipative small-scale $\eta$ and a large forcing scale $L$, for which the statistical moments of velocity increments depend only on the scale $r$ and energy dissipation per unit mass $\epsilon$. This self-similar mechanism gave the first quantitative interpretation of the well-known $k^{-5 / 3}$ energy spectrum of inertial turbulence, where $k=2 \pi / r$ is the wave number [8]. Although 


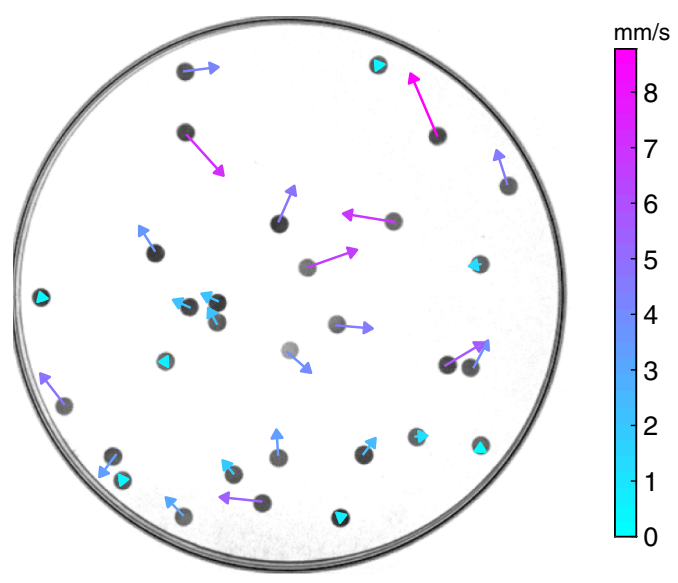

(a)

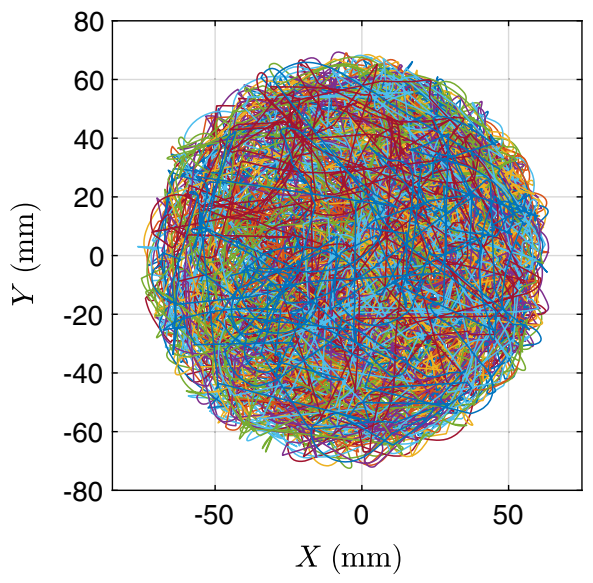

(b)

FIG. 1. (a) Top view of the experiment. Thirty self-propelled camphor disks move at an air-water interface. Arrows represent the instantaneous velocity direction, colored according to the velocity magnitude. A video is available as online Supplemental Material [23]. (b) Superposition of the trajectories of the 30 particles over a 5-min recording.

Kolmogorov's approach has been rapidly shown to fail predicting high-order moments (typically $n>4$ ) due to intermittency [9], it has so influenced the field that it is referred to as $\mathrm{K} 41$ phenomenology.

The analogy between active matter and turbulence first emerged from qualitative observations of mesoscale patterns (such as whirls, jets, and vortices) in dense biological suspensions [10], which seem ubiquitous in a wide variety of active systems [11] and are visually reminiscent of typical structures of inertial turbulence. On a more quantitative level, multiscale energy spectra were reported for a few dense active systems, for which a continuum approach allowed one to define a Eulerian flow field from the particles' dynamics $[2,12,13]$, and several studies investigated how active systems can be described as hydrodynamical flows based on generalized Navier-Stokes equations with tunable nonlinearity [2,13-16]. However, such descriptions were found to generally exhibit flow spectra with nonuniversal scaling exponents depending on model parameters. For instance, a recent numerical and analytical study suggests that while displaying multiscale energy spectrum, turbulence in active nematic systems falls into a universality class distinct from inertial turbulence, for which the energy is injected and dissipated at the same scale with no underlying cascade [17]. On the contrary, in a different class of active system made of small active spinners, experimental observations made on the generated fluid flow evidenced a kinetic energy spectrum compatible with Kolmogorov theory [18], thus fostering the analogy.

To date, most studies questioned such an analogy by focusing solely on the spatial correlations (or spectra in Fourier space) computed from snapshots of a Eulerian velocity field with such a field defined using the fluid if there is one, or as a continuous flow by local averaging over particles' velocities in dense systems. Yet, turbulent flows exhibit both temporal and spatial fluctuations, which are such that the timescales measured in the Lagrangian framework are related by a specific scaling to the length scales measured in the Eulerian framework [19]. Exploring the possible strong ties between active systems and inertial turbulence thus requires that we also look into the selfconsistency of possible analogies in both the Lagrangian and Eulerian frameworks. Overall, These different elements leave the question whether classes of active systems can be described in the inertial turbulence framework essentially open.

Finding such active systems exhibiting the same multiscale dynamics as observed in turbulence would not only benefit the future development of active systems but may also provide new insights into the long-standing description of turbulent flows. This is precisely the purpose of the present work to design and explore a synthetic active system displaying complex spatiotemporal dynamics amenable to combined Lagrangian and Eulerian characterization. The abiotic system considered is made of a dilute assembly of $\mathcal{O}(30)$ self-propelled camphor swimmers which generate their own spatiotemporal fluctuations when confined in a box [Fig. 1(a)]. It is therefore very different from the other systems described previously, as no continuous description can be employed. In light of classical statistical indicators from turbulence, we analyze the dynamics of the swimmers (rather than the one of the fluid in between them as was done in Ref. [18]).

Our experiments reveal striking similarities with inertial turbulence as both the Lagrangian and Eulerian dynamics of the active particles are found, to a large extent, indistinguishable from that of fluid tracers in turbulence. In particular, Eulerian statistics exhibit a well-identifiable inertial range of scales following remarkably classical K41 scalings of inertial turbulence with a turbulent Reynolds number compatible 
with the timescales measured in the Lagrangian framework. Here, the turbulent behavior is observed only on the statistics of the swimmers, as the subsurface fluid flow is found to be at most chaotic, so that the present system differs from other types of active turbulence as generated, for instance, by living matter.

\section{EXPERIMENTAL SETUP}

The scientific community has been prolific in the last decade in developing synthetic active particles and in investigating their individual and collective behaviors, with the goal to unveil generic physical properties of active matter [11]. Here, we consider macroscopic synthetic swimmers based on the historical realization of camphor boats [20]. More precisely, we use agar gel disks loaded with precipitated camphor [21], with radius $a=2.5 \mathrm{~mm}$ and height $h=0.6 \mathrm{~mm}$. When individually deposited at an air-water interface, the disks self-propel (with typical swim velocity $U_{s}$ in the order of $10 \mathrm{~mm} \mathrm{~s}^{-1}$ [22]) by a Marangoni effect arising from the camphor spreading at the interface. The individual hydrodynamical Reynolds number of such swimmers, $\operatorname{Re}_{p}=U_{s} a / \nu$ (with $\nu=1 \times 10^{-6} \mathrm{~m}^{2} \mathrm{~s}^{-1}$ the kinematic viscosity of water), is of the order of 25 , and no fluid turbulence is induced in the particles' wakes. Figure 1(a) shows a top view of our experiment, with 30 such self-propelled disks swimming on water in a 140-mmdiameter Petri dish filled with $1 \mathrm{~cm}$ of water. A video of the motion of the self-propelled disks is given as Supplemental Material [23]. The motion of the self-propelled disks is recorded at a frame rate of $30 \mathrm{fps}$ with a $1-\mathrm{Mpx}$ digital camera, and their motion is analyzed using classical 2D particle tracking to reconstruct the Lagrangian trajectories of each individual camphor disk. Simultaneous trajectories about 5-min long are retrieved for all particles. Figure 1(b) shows the superposition of the 30 trajectories simultaneously recorded, emphasizing that the available domain is explored ergodically. This is because great care is taken to adjust the level of water so that the surface remains horizontal at the edge of the vessel in order to prevent attraction or repulsion of the particles due to capillary forces. From the diameter of the particles image (35 pixels), the error on the particle position is estimated as $\delta X=0.5 / \sqrt{35} \leq 0.1$ pixels, a very small value as compared to the typical particle displacement $d X \simeq 5$ pixels between two frames. Even if this level of noise is small, velocity and acceleration of the camphor boats are computed by convolution with first and second derivatives of a Gaussian kernel [24] to filter small-scale noise.

In agreement with previous observations [25], we find a transition between a dilute and an interacting swimming regime. In the dilute regime, particles weakly affect each other, with individual trajectories essentially straight except for short collisionlike events or when a particle hits the boundaries. The interacting regime reveals, on the contrary, a much richer random collective dynamics with persistent high activity periods exhibiting large-scale correlations, emblematic of the concept of active matter. This transition can be seen, for instance, from the mean-square displacement (MSD) of the camphor boats as a function of time $t$ :

$$
\sigma_{\mathrm{MSD}}^{2}(t)=\left\langle\left(\vec{X}\left(t+t_{s}\right)-\vec{X}\left(t_{s}\right)\right)^{2}\right\rangle,
$$

where $\vec{X}$ is the instantaneous position of a camphor boat and $\langle\bullet\rangle$ an average over particles and starting time $t_{s}$ [see Fig. 2(a)]. For a small number of boats (typically $N_{p}<20$ ), the MSDs exhibit two trivial regimes: (i) At short times, the MSD grows ballistically $\left(\sigma_{\mathrm{MSD}}^{2} \propto t^{2}\right)$; (ii) at long times, the MSD oscillates around an asymptotic constant value. These regimes are consistent with a simple dynamics where particles essentially undergo a straight ballistic motion, with periodic reorientation at the boundaries due to the finite size of the experiment. For a large number of particles ( $\left.N_{p} \gtrsim 20\right)$, a third intermediate regime appears, where the MSD grows diffusively $\left(\sigma_{\mathrm{MSD}}^{2} \propto t\right)$. This regime is characteristic of a randomization of the individual dynamics, with a finite correlation timescale, as observed, for instance, for passive particles following a Langevin dynamics and also for tracers in inertial turbulence, which is known to exhibit at large scales a similar effective turbulent diffusive behavior $[26,27]$. Beyond the occurrence of this dilute-tointeracting transition, the present article aims to further explore the possible analogies between interacting active systems and inertial turbulence. The global behavior of the aforementioned transition for the collective dynamics of camphor boats has indeed already been addressed in previous studies [25], which have shown, for instance, that the transition occurs above a given threshold (setup dependent) of the total perimeter length $\ell_{p}=2 N_{p} \pi a$ and that the activity of the system then decreases (and eventually freezes for very dense systems). In our system, the dilute-to-interacting transition occurs around $N_{p} \approx 20$ (corresponding to a surface fraction of particles of $\phi_{s} \approx 2 \%$ and a total perimeter length $\ell_{p} \approx 300 \mathrm{~mm}$ ), while the system is observed to freeze for $N_{p} \gtrsim 60$. The decrease of the individual activity when increasing the number of particles is also revealed in Fig. 2(a) from the shift of the different curves in the ballistic regime at increasing $N_{p}$ as the MSD is related to the velocity variance, $\sigma_{v}^{2}=\left\langle v_{x}^{2}\right\rangle+\left\langle v_{y}^{2}\right\rangle$, by the relation $\log \sigma_{\mathrm{MSD}}^{2}=\log \sigma_{v}^{2}+2 \log t$.

Because the details of the transition are outside the scope of the present work, we rather focus on unveiling the rich collective and multiscale dynamics in the interacting regime $\left(N_{p}>20\right)$, which we characterize using statistical diagnoses borrowed from the turbulence community. As turbulence is a spatiotemporal phenomenon, correlation timescales extracted in the Lagrangian framework (following fluid particle trajectories) are directly related to length scales estimated in the Eulerian framework (from the 


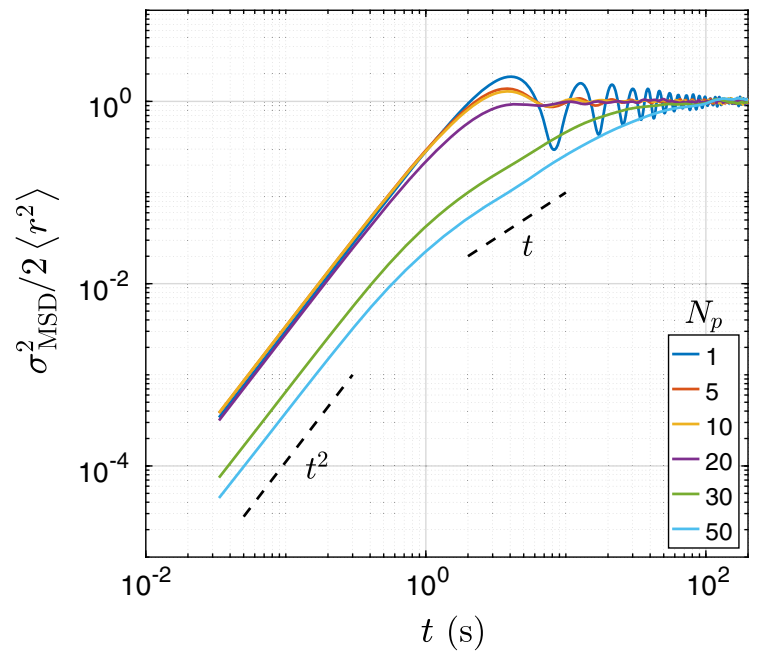

(a)

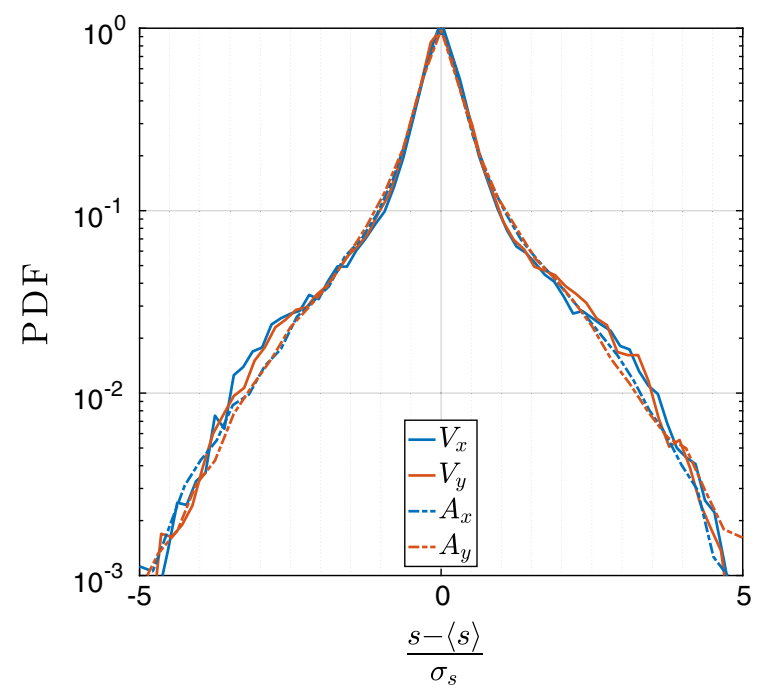

(b)

FIG. 2. (a) Normalized mean-square displacements $\left(\sigma_{\mathrm{MSD}}^{2} / 2\left\langle r^{2}\right\rangle\right.$, where $\left\langle r^{2}\right\rangle$ is the average square radial position) of the camphor boats for experiments with different number of particles $N_{p}$. At large times, the MSD oscillates around and eventually saturates to 1 , due to the finite size of the experiment. For small numbers of particles (typically $N_{p}<20$ ), the MSDs before saturation exhibit a dominant trivial ballistic motion (where the MSD grows as the square of time). For larger numbers of particles, an intermediate diffusivelike regime (where the MSD grows linearly with time) appears, revealing a nontrivial random dynamics with a finite correlation timescale. (b) Probability density functions of velocity and acceleration components. Because of the boundedness of the system, the mean velocity and acceleration components are zero. The standard deviation of the velocity components is $\sigma_{v_{x}}=\sigma_{v_{y}}=\left\langle v_{i}^{2}\right\rangle_{i=x, y}^{1 / 2}=12.1 \mathrm{~mm} \mathrm{~s}{ }^{-1}$ (corresponding to a root-mean-square velocity amplitude $\sigma_{|v|}=17.1 \mathrm{~mm} \mathrm{~s}^{-1}$ ); the standard deviation of acceleration components is $\sigma_{a_{x}}=\sigma_{a_{y}}=\left\langle a_{i}^{2}\right\rangle_{i=x, y}^{1 / 2}=33.2 \mathrm{~mm} \mathrm{~s}^{-2}$ (corresponding to a root-mean-square acceleration amplitude $\sigma_{|a|}=46.9 \mathrm{~mm} \mathrm{~s}^{-2}$ ).

analysis of the velocity field). We therefore study the statistics of the active particles in both the Lagrangian and Eulerian frameworks in order to explore possible quantitative analogies between the collective motion of the active disks and inertial turbulence. The results discussed in the sequel are obtained with the number of particles fixed at $N_{p}=30$, which corresponds to a typical situation with rich collective dynamics and strong individual activity.

\section{LAGRANGIAN DYNAMICS OF ACTIVE TURBULENCE}

In this section, we analyze the Lagrangian dynamics of the present active system at the light of inertial turbulence. As we see, characterization of relevant timescales allows us to determine an equivalent Reynolds number for our system, the consistency of which with Eulerian multiscale spectra is explored in the subsequent section.

\section{A. Single-time statistics}

Figure 2(b) shows the probability density functions (PDFs) of the components of the camphor boats' velocity $\left(v_{x}, v_{y}\right)$ and acceleration $\left(a_{x}, a_{y}\right)$ where the variables are centered-although the mean velocity and acceleration are vanishingly small due to confinement of the system-and reduced so that the shapes of the curves can be compared. Several points can be noted: (i) The PDFs are identical for the two components, revealing the isotropy of the dynamics; (ii) the PDFs of velocity and acceleration are identical. In the context of inertial turbulence, such a result would be interpreted as the absence of internal intermittency in the Lagrangian framework [28]. (iii) The PDFs are not Gaussian (but without any stretched tail), a feature commonly reported in 2D fluid turbulence [29-31]. Because of the observed isotropy, the statistics are further investigated in the sequel considering only the $x$ component of the motion.

\section{B. Two-times statistics}

In addition to the mean-square displacement previously discussed, two of the most fundamental indicators used to explore the multiscale Lagrangian dynamics of turbulence are the autocorrelation functions of the velocity and acceleration components:

$$
\begin{aligned}
& R_{v_{i} v_{i}}(t)=\left\langle v_{i}\left(t_{0}\right) v_{i}\left(t+t_{s}\right)\right\rangle /\left\langle v_{i}^{2}\right\rangle, \\
& R_{a_{i} a_{i}}(t)=\left\langle a_{i}\left(t_{0}\right) a_{i}\left(t+t_{s}\right)\right\rangle /\left\langle a_{i}^{2}\right\rangle,
\end{aligned}
$$

where again, averages are taken over particles and starting time $t_{s}$. These functions are important statistical tools 


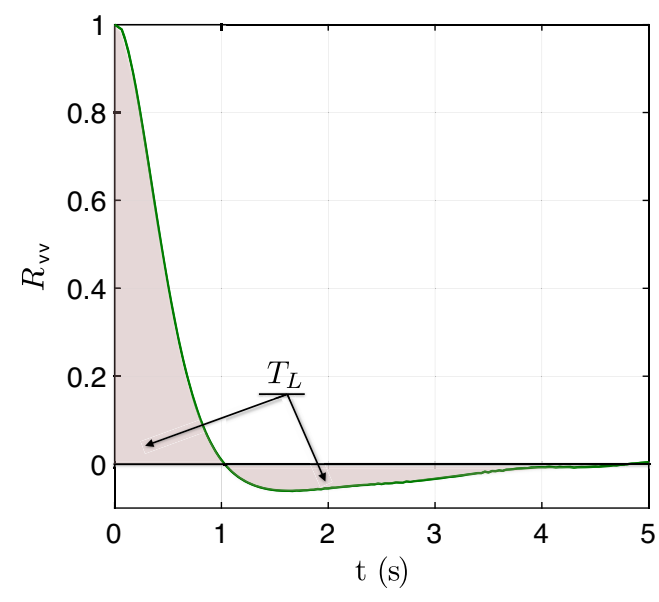

(a)

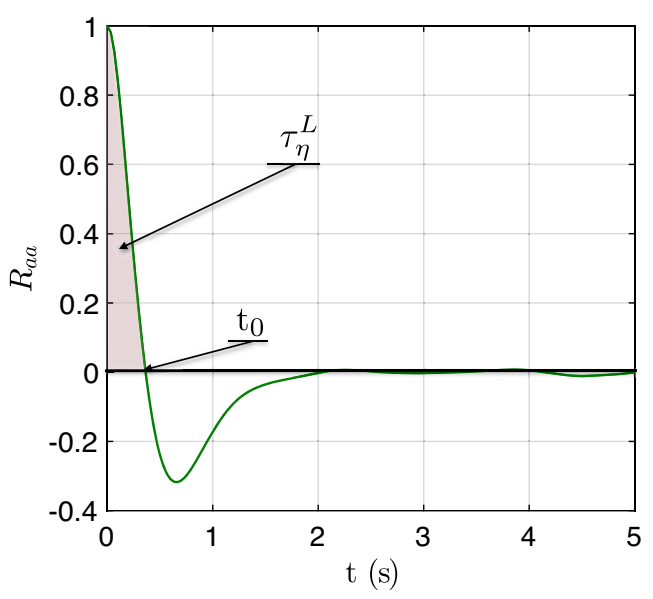

(b)

FIG. 3. Single particle-two-times Lagrangian statistics. (a) Lagrangian autocorrelation function $R_{\nu \nu}$ of velocity component $v_{x}$. The light red colored area represents the estimate of the Lagrangian correlation timescale $T_{L}$. (b) Lagrangian autocorrelation function $R_{a a}$ of acceleration component $a_{x}$. $t_{0}$ corresponds to the shortest time such as $R_{a a}\left(t_{0}\right)=0$. The light red colored area represents the estimate of the Lagrangian dissipative timescale $\tau_{\eta}^{L}$.

allowing us to quantify the multiscale temporal dynamics of turbulence in terms of a hierarchy of relevant dynamical regimes: the Lagrangian dissipative regime at small scales where the dynamics is smooth and regularized by viscosity, the Lagrangian inertial regime at intermediate scales where the dynamics is rough and correlated, and the Lagrangian uncorrelated regime at large scales. The existence of an extended range of inertial scales is one of the most important features of inertial turbulence. In classical turbulence, two important timescales are defined based on the correlation properties of velocity and acceleration, which delimit the extent of the inertial range: (i) the integral Lagrangian timescale, which we define here as $T_{L}=$ $\int_{0}^{\infty}\left|R_{v v}\right| d t$ (absolute values are requested here because the particle position is bounded [32]) and (ii) the dissipative Lagrangian timescale $\tau_{\eta}^{L}$, traditionally taken as [33] $\tau_{\eta}^{L}=$ $\int_{0}^{t_{0}} R_{a a}(t) d t \quad$ [with $t_{0}$ the shortest time for which $R_{a a}\left(t_{0}\right)=0$ ]. The dynamics occurring at scales smaller than $\tau_{\eta}^{L}$ is then referred to as dissipative, the dynamics at scales larger than $T_{L}$ is referred to as uncorrelated. and the dynamics at scales intermediate between $\tau_{\eta}^{L}$ and $T_{L}$ is referred to as inertial. $T_{L}$ and $\tau_{\eta}^{L}$ then define the Lagrangian Reynolds number [27] $\operatorname{Re}^{L}=\left(T_{L} / \tau_{\eta}^{L}\right)^{2}$ which characterizes the extent of the Lagrangian inertial range of timescales.

Here we analyze the multiscale random Lagrangian dynamics of the active camphor disks within the same framework of inertial turbulence just described. Figures 3(a) and 3(b) show the velocity and acceleration autocorrelation functions for the active camphor disks. The corresponding integral and dissipative Lagrangian timescales are $T_{L} \approx 0.6 \mathrm{~s}$ and $\tau_{\eta}^{L} \approx 0.2 \mathrm{~s}$, leading to an equivalent Lagrangian Reynolds number $\operatorname{Re}^{L} \approx 9$. Although this number may seem small at first sight, the Lagrangian Reynolds number is known in inertial turbulence to be significantly smaller than the classical integral Eulerian Reynolds number $\operatorname{Re}^{E}=\left(L_{E} / \eta\right)^{4 / 3}$, where $L_{E}$ is the Eulerian (spatial) correlation length scale of the velocity fluctuations. Numerical simulations of inertial turbulence show that $\mathrm{Re}^{E}$ and $\mathrm{Re}^{L}$ are empirically related [27] by $\operatorname{Re}^{E} \approx 88.6 \mathrm{Re}^{L^{0.61}}$. In the inertial turbulence context, a Lagrangian Reynolds number $\operatorname{Re}^{L} \approx 9$ is therefore equivalent to an integral Reynolds number $\operatorname{Re}^{E} \approx 340$. To fix these ideas, such conditions would correspond to a moderate turbulence, such as generated in a large-scale (metric size test section) wind tunnel blowing at $3 \mathrm{~m} \mathrm{~s}^{-1}$ downstream a passive grid with $7-\mathrm{cm}$ mesh size $[34,35]$.

Overall, our active system of small (millimetric) camphor boats shows Lagrangian temporal signatures which are consistent with expectations for a typical inertial turbulence system. Based on this comparable multiscale random dynamics, it is possible to determine the integral Reynolds number $\operatorname{Re}^{E} \approx 340$ of the equivalent fluid system, which suggests a moderate turbulence regime. For a meaningful analogy to hold between the camphor interfacial swimmers and classical turbulence, it is mandatory that the spatial multiscale dynamics of our active system be consistent with expected signatures in the very same turbulence regime. To that aim, we now turn to the study of Eulerian properties.

\section{EULERIAN DYNAMICS OF ACTIVE TURBULENCE}

In this section, we investigate the Eulerian multiscale dynamics of the camphor disks by considering the spatial correlations between their instantaneous velocity across the 
system rather than their temporal correlations along individual disks' trajectories, as in the previous section. According to the results of the Lagrangian dynamics, to match the properties of an equivalent inertial turbulence system $\left(\operatorname{Re}^{E} \approx 340 ; R_{\lambda} \approx 70\right)$, the camphor swimmers dynamics should exhibit a narrow, but still visible inertial range of spatial scales. In particular, the second-order Eulerian structure function $S_{2}^{E}$ would exhibit a Kolmogorovian scaling $\left[S_{2}^{E}(r) \propto r^{2 / 3}\right]$ over slightly less than one decade of scales $r$ (or equivalently, the power spectral density would exhibit a narrow range of scales in Fourier space with a $k^{-5 / 3}$ regime). Given the small number

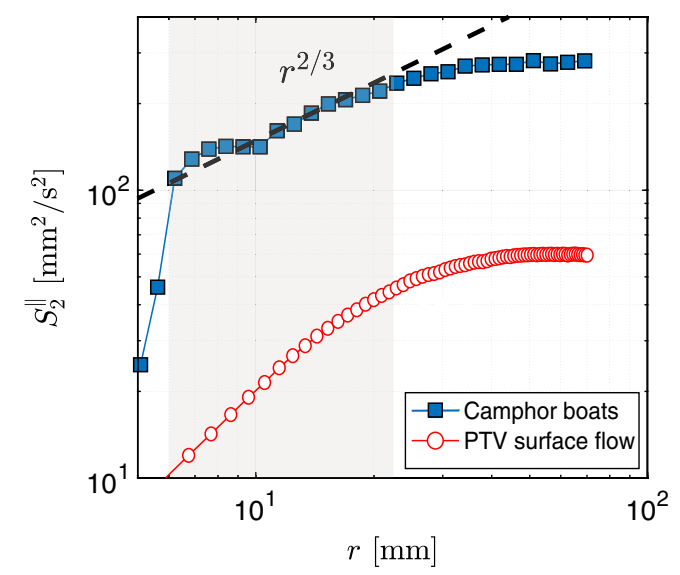

(a)

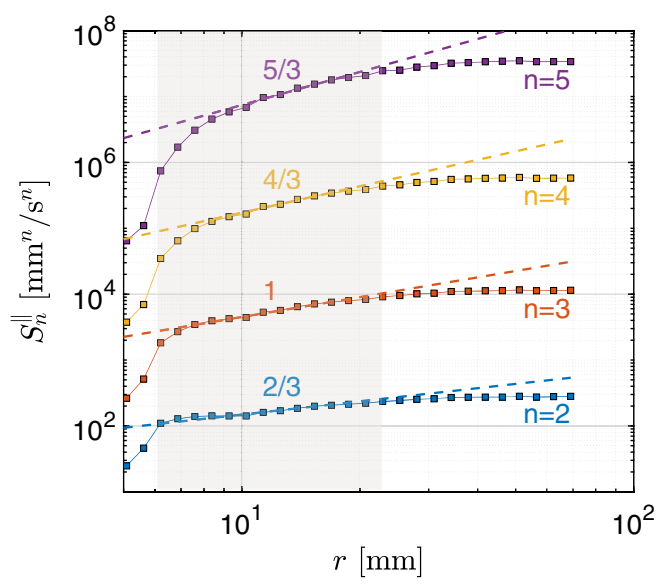

(c) of active particles in the system, it is impossible to define a Eulerian velocity field by binning particles velocities in space as was done in Wensink and Löwen [12] for dense suspensions. However, such a field is not required for the computation of Eulerian structure functions of the velocity differences:

$$
S_{p}^{\|}(r)=\left\langle\left|\frac{\left[\vec{v}_{i}(t)-\vec{v}_{j}(t)\right] \cdot \vec{r}_{i j}}{r_{i j}}\right|^{p}\right\rangle
$$

where $r$ represents a bin of spatial scales, and the average is taken over all pairs $(i, j)$ of particles with separation

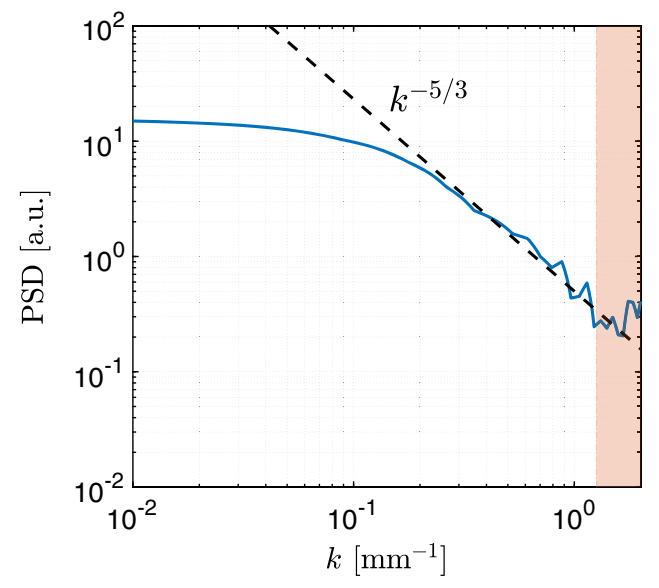

(b)

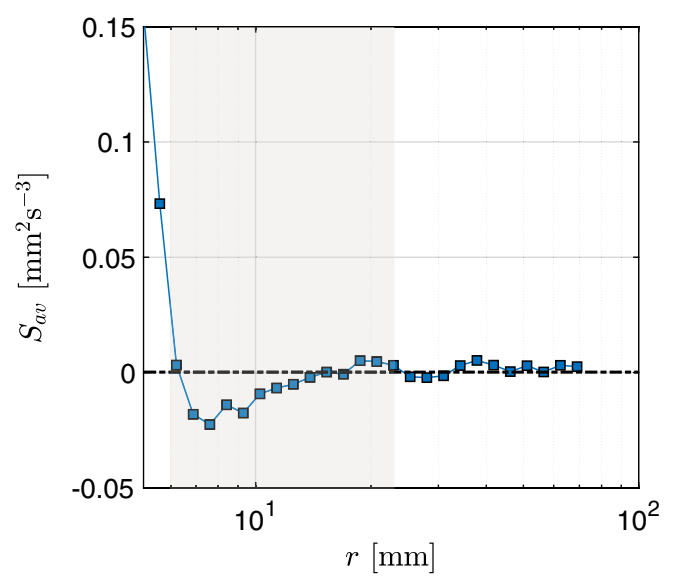

(d)

FIG. 4. Two particles_-single-time Eulerian statistics. (a) ( $\square$ ) Second-order longitudinal structure function $S_{2}^{\|}$as a function of interparticle separation $r$. The dashed line represents the K41 prediction for inertial turbulence $S_{2}^{\|} \propto r^{2 / 3}$. (o) $S_{2}^{\|}(r)$ for the fluid flow at the surface computed from the velocity field measured by particle tracking velocimetry (PTV) using 200- $\mu$ m floating tracers. (b) Power spectral density obtained as the Fourier transform of the Eulerian correlation function of the camphor boats' velocity. The dashed line represents the K41 prediction for inertial turbulence. The light red area on the right represents wave numbers corresponding to scales smaller that the particles' diameter. The Kolmogorov spectrum extends over almost one decade of scales, down to scales of the order of the particles' diameter. (c) High-order longitudinal structure functions $S_{n}^{\|}$(for $n \leq 5$ ). Dashed lines indicate the corresponding nonintermittent K41 predictions $\left(S_{n}^{\|} \propto r^{n / 3}\right)$. The light gray area qualitatively indicates the corresponding inertial range for which K41 scalings hold. (d) Crossed acceleration-velocity Eulerian structure function, whose amplitude in inertial turbulence is twice the energy flux, and the sign indicates the direction (direct or inverse) of the energy cascade: The negative sign here points toward a direct (from large- to small-scale) scenario. 
$r_{i j}$ within that bin. Figure 4(a) shows the longitudinal second-order Eulerian structure function $S_{2}^{\|}(r)$ as a function of the separation between the active particles. At small scales, $S_{2}^{\|}(r)$ vanishes for distances approaching the disk diameter $(5 \mathrm{~mm})$, as particles cannot interpenetrate. For large distances, $S_{2}^{\|}(r)$ tend to a constant asymptotic value $S_{2}^{\| \infty} \approx 290 \mathrm{~mm}^{2} \mathrm{~s}^{-2}$ (which corresponds to $\sigma_{|v|}^{2}$, the variance of the velocity module) as expected for uncorrelated particles. At intermediate separations, a range of scales is observed, where $S_{2}^{\|}(r) \propto r^{2 / 3}$, reminiscent of a Kolmogorovian inertial range. This Kolmogorovian inertial range of active turbulence is also visible in the Eulerian energy spectrum (estimated as the Fourier transform of the Eulerian correlation function)

$$
R_{v v}^{E}(r)=1-\frac{S_{2}^{\|}(r)}{\sigma_{|v|}^{2}}
$$

shown in Fig. 3(b), which exhibits a noticeable $k^{-5 / 3}$ regime down to wave numbers corresponding to the particle diameter. We also estimate higher-order Eulerian structure functions $S_{n}^{\|}(r)$ [Fig. 3(c)] which are found, within the range of inertial scales just identified, to follow nonintermittent $\mathrm{K} 41$ inertial scalings $S_{n}^{\|}(r) \propto r^{n / 3}$. Such a result is consistent with the absence of internal intermittency that is observed in the Lagrangian framework when investigating velocity and acceleration PDFs of the particles.

From these results, one may think that the Marangoni surfers are randomly forcing the flow at the surface so that a classical turbulent flow takes places. In order to check that it is not the case, we compute the second-order structure function from 22000 trajectories obtained by tracking $\mathcal{O}(500)$ small passive floating tracers freely advected in between the camphor particles. Figure 4(a) shows that the structure function for the fluid flow is much smaller than the one for the active particles, indicating that the forcing at the surface is not very efficient, except in the vicinity of swimmers. This inefficiency is the reason why no inertial regime with an exponent $2 / 3$ is observed for the fluid in the intermediate range of scales. Indeed, the fluid displays a smoother behavior than the particles with no clear scaling, so that the fluid flow may be regarded as chaotic, but not as turbulent.

\section{DISCUSSION}

Altogether, our results concerning the collective dynamics of the camphor boats show that it shares remarkable quantitative analogies with turbulence, from both the Lagrangian and the Eulerian points of view: Indeed, the nonintermittent scalings that we find for the high-order Eulerian structure functions are reminiscent of the inverse cascade regime in 2D fluid turbulence $[36,37]$. Considering that in active matter, energy is primarily injected at the particle level, it is therefore tempting to link the absence of intermittency to the existence of an inverse energy cascade of active turbulence. Figure 4(d) represents the energy flux across scales, as it could be estimated for inertial turbulence, based on the crossed velocity-acceleration Eulerian structure function [38] $S_{a v}^{E}(r)=\left\langle\delta_{r} \vec{a} \cdot \delta_{r} \vec{v}\right\rangle$ of the active particles. The negative value of $S_{a v}^{E}$ over the range of scales previously identified as inertial reveals that the turbulentlike energy cascade in the present system is actually not inverse, but direct (energy flows from large to small scales, as in 3D inertial turbulence, for which $S_{a v}^{E} \sim-2 \epsilon$ ). The multiscale Eulerian dynamics is therefore not associated here with an upward energy flux originating from individual particles as energy source. Rather, it originates from large-scale interactions, forcing down to smaller scales the collective dynamics of the particles. In the present situation, the chemical background of the dissolved camphor left behind the active particles could be the vector of such longrange interactions. Indeed, given the small Reynolds number of the particles $\operatorname{Re}_{p} \sim 25$, no direct inertial cascade of turbulence can take place in the fluid forced by the swimmers. This is confirmed from the investigation of the motion of small tracers floating on the surface [lowest curve in Fig. 4(a)] that proves that the surface flow is not turbulent, although slightly agitated. To support the scenario of a chemically mediated interaction, it would be enlightening in future studies to monitor the chemical camphor background at the same time as the particles' dynamics.

In the context of active matter, our results are important as they show the first example of active turbulence with inertial range dynamics quantitatively similar to a direct energy cascade in K41 inertial turbulence. To which extent this connection between active and inertial turbulence can be extended to other active systems, in particular, with long-range interactions (chemical, hormonal, visual, etc.), is an important opening of this work.

In the context of inertial turbulence, our results are also important as they provide a simple experimental model of a nonintermittent direct cascade of energy. The absence of intermittency may be related to the absence of a dissipative cutoff in the energy spectrum and structure functions, which follow K41 scalings down to scales of the order of the particle diameter. The small-scale dynamics is therefore limited here by particle-particle collisions, but does not exhibit any effective viscosity behavior. In 2D turbulence, the absence of intermittency in the inverse cascade tends indeed to be associated with the idea that as energy flows upwards in scales, the effect of viscous dissipation at small scales may be disregarded [37]. In this respect, the parallel between active and inertial turbulence may help in the future to better understand the origin and the modeling of intermittency, which remains one of the biggest mysteries of turbulence in fluids. 


\section{ACKNOWLEDGMENTS}

The authors thank Clément Gouiller for his help during the particle tracking experiments of passive tracers at the surface of the flow. This collaborative work is supported by the French research programs ANR-16CE30-0028, Laboratoire d'Excellence (LABEX) Institute for Multiscale Science and Technology (iMUST) (ANR10-LABX-0064), and Initiative d'excellence de Lyon (IDEXLYON) of the University of Lyon in the framework of the Programme Investissements d'Avenir (ANR-16IDEX-0005).

[1] C. Dombrowski, L. Cisneros, S. Chatkaew, R. E. Goldstein, and J. O. Kessler, Self-Concentration and Large-Scale Coherence in Bacterial Dynamics, Phys. Rev. Lett. 93, 098103 (2004).

[2] H. H. Wensink, J. Dunkel, S. Heidenreich, K. Drescher, R. E. Goldstein, H. Löwen, and J. M. Yeomans, Meso-Scale Turbulence in Living Fluids, Proc. Natl. Acad. Sci. U.S.A. 109, 14308 (2012).

[3] M. C. Marchetti, J. F. Joanny, S. Ramaswamy, T. B. Liverpool, J. Prost, M. Rao, and R. A. Simha, Hydrodynamics of Soft Active Matter, Rev. Mod. Phys. 85, 1143 (2013).

[4] A. Doostmohammadi, T. N. Shendruk, K. Thijssen, and J. M. Yeomans, Onset of Meso-Scale Turbulence in Living Fluids, Nat. Commun. 8, 15326 (2016).

[5] P. Guillamat, J. Ignés-Mullol, and F. Sagués, Taming Active Turbulence with Patterned Soft Interfaces, Nat. Commun. 8, 564 (2017).

[6] K.-T. Wu, J. B. Hishamunda, D. T. N. Chen, S. J. DeCamp, Y.-W. Chang, A. Fernández-Nieves, S. Fraden, and Z. Dogic, Transition from Turbulent to Coherent Flows in Confined Three-Dimensional Active Gluids, Science 355, eaal1979 (2017).

[7] A. Kolmogorov, The Local Structure of Turbulence in Incompressible Viscous Fluid for Very Large Reynolds Numbers, Dokl. Akad. Nauk SSSR 30, 301 (1941) [Proc. R. Soc. A 434, 9 (1991)].

[8] In the framework of Kolmogorov's theory, Eulerian velocity structure functions defined as the statistical moments of the velocity increments $\delta_{r} \mathbf{u}=\mathbf{u}(\mathbf{x}+\mathbf{r})-\mathbf{u}(\mathbf{x})$ between points of the flow separated by a distance $r=|\mathbf{r}|$ are expected to scale as $S_{n}(r)=\left\langle\left|\delta_{r} \mathbf{u}\right|^{n}\right\rangle \propto(\epsilon r)^{n / 3}$ for $r$ within the inertial range. The $k^{-5 / 3}$ energy spectrum of inertial turbulence is the Fourier space equivalent to $S_{2}(r) \propto(\epsilon r)^{2 / 3}$, with $k=2 \pi / r$.

[9] U. Frisch, Turbulence: The Legacy of A. N. Kolmogorov (Cambridge University Press, Cambridge, England, 1995).

[10] N. H. Mendelson, A. Bourque, K. Wilkening, K. R. Anderson, and J. C. Watkins, Organized Cell Swimming Motions in Bacillus subtilis Colonies: Patterns of ShortLived Whirls and Jets, J. Bacteriol. 181, 600 (1999).

[11] C. Bechinger, R. Di Leonardo, H. Löwen, C. Reichhardt, G. Volpe, and G. Volpe, Active Particles in Complex and Crowded Environments, Rev. Mod. Phys. 88, 045006 (2016).

[12] H. H. Wensink and H. Löwen, Emergent States in Dense Systems of Active Rods: From Swarming to Turbulence, J. Phys. Condens. Matter 24, 464130 (2012).

[13] X. Qiu, L. Ding, Y. Huang, M. Chen, Z. Lu, Y. Liu, and Q. Zhou, Intermittency Measurement in Two-Dimensional Bacterial Turbulence, Phys. Rev. E 93, 062226 (2016).

[14] J. Toner and Y. Tu, Long-Range Order in a TwoDimensional Dynamical XY Model: How Birds Fly Together, Phys. Rev. Lett. 75, 4326 (1995).

[15] V. Bratanov, F. Jenko, E. Frey, and A. J. Smits, New Class of Turbulence in Active Fluids, Proc. Natl. Acad. Sci. U.S.A. 112, 15048 (2015).

[16] M. James and M. Wilczek, Vortex Dynamics and Lagrangian Statistics in a Model for Active Turbulence, Eur. Phys. J. E 41, 21 (2018).

[17] R. Alert, J.-F. Joanny, and J. Casademunt, Universal Scaling of Active Nematic Turbulence, arXiv:1906.04757.

[18] G. Kokot, S. Das, R. G. Winkler, G. Gompper, I. S. Aranson, and A. Snezhko, Active Turbulence in a Gas of Self-Assembled Spinners, Proc. Natl. Acad. Sci. U.S.A. 114, 12870 (2017) [https://www.pnas.org/content/114/49/12870 .full.pdf].

[19] H. Tennekes and J. L. Lumley, A First Course in Turbulence (MIT, Canbridge, MA, 1972).

[20] L. Rayleigh, Measurements of the Amount of Oil Necessary in Order to Check the Motions of Camphor upon Water, Proc. R. Soc. 47, 364 (1889), https://www.jstor.org/stable/ 114951.

[21] S. Soh, K. J. M. Bishop, and B. A. Grzybowski, Dynamic Self-Assembly in Ensembles of Camphor Boats, J. Phys. Chem. B 112, 10848 (2008).

[22] D. Boniface, C. Cottin-Bizonne, R. Kervil, C. Ybert, and F. Detcheverry, Self-Propulsion of Symmetric Chemically Active Particles: Point-Source Model and Experiments on Camphor Disks, Phys. Rev. E 99, 062605 (2019).

[23] See Supplemental Material at http://link.aps.org/ supplemental/10.1103/PhysRevX.10.021065 for temporal dynamics of 30 Marangoni surfers loaded with camphor. The scene is recorded from above at $30 \mathrm{fps}$ and lit from bellow with a LED panel.

[24] N. Mordant, A. M. Crawford, and E. Bodenschatz, Experimental Lagrangian Acceleration Probability Density Function Measurement, Physica (Amsterdam) 193D, 245 (2004).

[25] N. J. Suematsu, K. Tateno, S. Nakata, and H. Nishimori, Synchronized Intermittent Motion Induced by the Interaction between Camphor Disks, J. Phys. Soc. Jpn. 84, 034802 (2015).

[26] G. I. Taylor, Diffusion by Continuous Movements, Proc. London Math. Soc. s2-20, 196 (1922).

[27] B. L. Sawford, Reynolds Number Effects in Lagrangian Stochastic Models of Turbulent Dispersion, Phys. Fluids A 3, 1577 (1991).

[28] In the context of 3D (homogeneous and isotropic) turbulence, the velocity PDF is close to Gaussian while the acceleration PDF is strongly non-Gaussian so that the PDF of velocity increments $\delta_{\tau} v_{i}=v_{i}(t+\tau)-v_{i}(t)(i=x, y)$ strongly depends on the time increment $\tau$. As acceleration is strongly linked to local dissipation, this behavior is a 
consequence of internal intermittency (in contrast to external intermittency, which refers to a bursting temporal dynamics often observed at a turbulent-nonturbulent interface).

[29] A. Bracco, J. LaCasce, C. Pasquero, and A. Provenzale, The Velocity Distribution of Barotropic Turbulence, Phys. Fluids 12, 2478 (2000).

[30] C. Pasquero, A. Provenzale, and A. Babiano, Parameterization of Dispersion in Two-Dimensional Turbulence, J. Fluid Mech. 439, 279 (2001).

[31] Y. K. Tsang, Nonuniversal Velocity Probability Densities in Two-Dimensional Turbulence: The Effect of Large-Scale Dissipation, Phys. Fluids 22, 115102 (2010).

[32] Because the velocity signal $\vec{v}(t)=d \vec{X} / d t$ is statistically stationary with bounded position $\vec{X}(t)$, one has $\int_{0}^{\infty} R_{v v}\left(t^{\prime}\right) d t^{\prime}=0$. This result is obtained from the equality $d\left\langle[\vec{X}(t)-\vec{X}(0)]^{2}\right\rangle / d t=2 \int_{0}^{t} R_{v v}\left(t^{\prime}\right) d t^{\prime}$.

[33] E. Calzavarini, R. Volk, M. Bourgoin, E. Leveque, J. F. Pinton, and F. Toschi, Acceleration Statistics of Finite-Sized
Particles in Turbulent Flow: The Role of Faxen Forces, J. Fluid Mech. 630, 179 (2009).

[34] Such a flow corresponds to an integral Reynolds number $\operatorname{Re}^{E} \approx 340$ and a Taylor-scale Reynolds number $\operatorname{Re}_{\lambda} \approx 70$ estimated through the relation $R_{\lambda} \approx\left(15 \mathrm{Re}^{E}\right)^{1 / 2}$.

[35] R. Monchaux, M. Bourgoin, and A. Cartellier, Preferential Concentration of Heavy Particles: A Voronoi Analysis, Phys. Fluids 22, 103304 (2010).

[36] T. Dubos, A. Babiano, J. Paret, and P. Tabeling, Intermittency and Coherent Structures in the Two-Dimensional Inverse Energy Cascade: Comparing Numerical and Laboratory Experiments, Phys. Rev. E 64, 036302 (2001).

[37] G. Boffetta, A. Celani, and M. Vergassola, Inverse Energy Cascade in Two-Dimensional Turbulence: Deviations from Gaussian Behavior, Phys. Rev. E 61, 29(R) (2000).

[38] J. Jucha, H. Xu, A. Pumir, and E. Bodenschatz, TimeReversal-Symmetry Breaking in Turbulence, Phys. Rev. Lett. 113, 054501 (2014). 